\title{
Basic Study on Active Changes in Biological Function of Mouse Liver Graft in Cold Storage after Low-Dose X-Irradiation
}

\author{
Takahiro Kataoka, Masaaki Yoshimoto, Shinya Nakagawa, Yuko Mizuguchi, Takehito Taguchi, \\ and Kiyonori Yamaoka*
}

Graduate School of Health Sciences, Okayama University, 5-1 Shikata-cho, 2-chome, Okayama 700-8558, Japan

Received 22 January, 2009; Accepted 27 April, 2009

\begin{abstract}
Summary We previously reported that low-dose $\mathrm{X}$-irradiation alleviates ischemia-reperfusion injury such as mouse paw edema. In this study, we examined active changes in the biological function of mouse liver grafts in cold storage after low-dose $X$-irradiation. Mouse livers were sham-irradiated or were irradiated with $0.25,0.5,1.0$, or $5.0 \mathrm{~Gy}$ of $\mathrm{X}$-ray and stored for 4,8 , 24 , or $48 \mathrm{~h}$ in preservation or saline solution. The results show that storage for $24 \mathrm{~h}$ in saline solution after $0.5 \mathrm{~Gy}$ irradiation significantly increased the activity of superoxide dismutase (SOD) and catalase. Following storage for 4,8 , or $48 \mathrm{~h}$ in preservation solution, lipid peroxide levels of the $0.5 \mathrm{~Gy}$ irradiated group were significantly lower than those of the sham irradiated group. Following storage for $24 \mathrm{~h}$ in preservation solution, the activity of SOD and catalase of the 1.0 Gy irradiated group were significantly higher than those of the sham irradiated group. Hepatocytes stored in saline solution were vacuolated. However, no vacuole formation was observed in hepatocytes stored in preservation solution. These findings suggest that low-dose irradiation significantly activates antioxidative functions of liver grafts. Moreover, the dose at which enhancement of antioxidative function occurs in livers stored in preservation solution, which contains glutathione, is significantly higher than that in saline solution.
\end{abstract}

Key Words: organ transplantation, low-dose irradiation, antioxidative function, hepatopathy

\section{Introduction}

Clinical liver transplant is now widely applied for the treatment of end-stage liver disease. About 11,000 transplants are done annually worldwide. Patient survival at one year and five years is $85-90 \%$ and $75-80 \%$, and graft survival is $80-85 \%$ and $70-75 \%$, respectively [1]. Ischemiareperfusion injury is an inevitable result of liver transplantation and is closely related to the development of primary graft non-function and primary graft dysfunction [2]. Both conditions are associated with high rates of morbidity and mortality. In addition, ischemia-reperfusion injury increases

*To whom correspondence should be addressed.

Tel/Fax: +81-86-235-6852

E-mail: yamaoka@md.okayama-u.ac.jp the incidence of subsequent graft rejection [3]. Livers are stored in a cold $\left(4^{\circ} \mathrm{C}\right)$ solution, such as Collins' solution or University of Wisconsin (UW) solution until transplantation. Canine livers are stored in UW solution for 24, 30, 36, or $48 \mathrm{~h}$ and transplanted animals that survive beyond 5 days after transplantation have excellent graft functions [4].

It is well known that ischemia-reperfusion injury is attributable to reactive oxygen species (ROS). Transient hypoxia increases hypoxanthine and xanthine in cells. The superoxide radical generated by xanthine oxidase (XOD) contributes to the development of ischemia-reperfusion injury $[5,6]$, and treatment with superoxide dismutase (SOD) shows beneficial effects on rat graft liver [7]. These results indicate that SOD plays an important role in the inhibition of ischemia-reperfusion injury after liver transplantation.

Low-dose irradiation increases endogenous antioxidants 
in animal tissue. It has been reported that antioxidants such as SOD [8-11], glutathione peroxidase (GPx) [9, 11], glutathione reductase (GR) [12], glutathione [11, 12], catalase [11], and thioredoxin [11] are activated and/or induced by low-dose irradiation and that they contribute to the inhibition of oxidative injury. Furthermore, we previously reported inhibitory effects of prior low-dose X-irradiation on ischemia-reperfusion injury in mouse paw [13]. These findings suggested that low-dose irradiation enhances antioxidative function and alleviates ischemia-reperfusion injury. Most of these studies have reported that activation of antioxidative function is induced by cell or whole body irradiation.

Activation of antioxidative function in grafts could potentially inhibit ischemia-reperfusion injury after transplantation. However, there have been no reports of changes in the antioxidative function of grafts following low-dose irradiation after exenteration. In the present study, we examined changes in antioxidative function and hepatocyte histology induced in extirpated mouse liver by low-dose $\mathrm{X}$-irradiation. Antioxidative functions such as lipid peroxide levels, total glutathione content, the activity of SOD and catalase were investigated as well as histological changes in the livers under various conditions of irradiation and storage.

\section{Materials and Methods}

\section{Animals, liver irradiation and storage}

Male BALB/c mice (body weight of about $25 \mathrm{~g}$, age; seven to eight weeks) were kept in an air-conditioned room (temperature: $20^{\circ} \mathrm{C}$, humidity, $60 \%$ ) at the Department of Animal Resources, the Advanced Science Research Center, Okayama University. Livers were perfused with a physiological saline solution under ether anesthesia before the livers were harvested and stored for $4,8,24$, or $48 \mathrm{~h}$ in preservation solution (Via Span; Bristol-Myars Squlbb Co.), at $4^{\circ} \mathrm{C}$ or in physiological saline solution (saline solution, $\mathrm{pH} 7.8$ at $4^{\circ} \mathrm{C}$ ). The preservation solution was used as a model for UW solution. Prior to storage in these solutions, the extirpated livers were irradiated at a dose of $0.25,0.5$, 1.0 , or $5.0 \mathrm{~Gy}(3.0 \mathrm{~Gy} / \mathrm{min})$ using an X-ray generator (Hitachi MBR-1505R2, voltage; $150 \mathrm{kV}$, ampere; $20 \mathrm{~mA}$, filters; $\mathrm{Cu}: \mathrm{Al}=0.5 \mathrm{~mm}: 0.2 \mathrm{~mm}$ ) at room temperature. The livers were stored in the solutions immediately after irradiation. At the end of this period of storage, livers were preserved at $-80^{\circ} \mathrm{C}$ until biochemical assay. Liver sections were fixed in $10 \%$ neutral-buffered formalin for histological observation. For comparison, the livers of a control group were sham irradiated immediately after liver exenteration and stored at $-80^{\circ} \mathrm{C}$ until biochemical assay. The study protocol was in accordance with the animal experimental guidelines of Okayama University.

\section{Biochemical assays}

Lipid peroxide (malondialdehyde (MDA)) levels were assayed using Bioxytech LPO-586 ${ }^{\mathrm{TM}}$ assay kit (OXIS Health Products, Inc., OR). Briefly, livers were homogenized in $20 \mathrm{mM}$ phosphate buffer (PBS; pH 7.4) on ice. Prior to homogenization, $10 \mu \mathrm{L}$ of $0.5 \mathrm{M}$ butylated hydroxytoluene in acetonitrile were added per $1 \mathrm{~mL}$ of tissue homogenate. After homogenization, the homogenate was centrifuged at $15,000 \times \mathrm{g}$, for $10 \mathrm{~min}$ at $4^{\circ} \mathrm{C}$ and the supernatant was used for assay. The MDA assay is based on the reaction of a chromogenic reagent, $\mathrm{N}$-methyl-2-phenylidole, with MDA at $45^{\circ} \mathrm{C}$. The optical density of the colored products was read at $586 \mathrm{~nm}$ in a spectrophotometer. The protein content was measured by the Bradford method, using Protein Quantification Kit-Rapid (Dojindo Molecular Technologies, Inc., Kumamoto, Japan) [14].

Total glutathione content was measured using the Bioxytech GSH- $420^{\mathrm{TM}}$ assay kit (OXIS Health Products). Briefly, livers were suspended in $10 \mathrm{mM}$ phosphate buffer ( $\mathrm{pH} 7.4$ ), mixed with ice-cold $7.5 \%$ trichloroacetic acid solution and then homogenized. The homogenates were centrifuged at $3,000 \times g$ for $10 \mathrm{~min}$. The supernatant was used for the assay. Total glutathione content was measured at $420 \mathrm{~nm}$ using a spectrophotometer. This assay is based on the formation of a chromophoric thione the absorbance of which, measured at $420 \mathrm{~nm}$, is directly proportional to the total glutathione concentration.

Mouse liver was homogenized in a $1 \mathrm{M}$ Tris-HCl buffer containing $5 \mathrm{mM}$ ethylendiaminetetraacetic acid (EDTA) $(\mathrm{pH} 7.4)$ on ice. The homogenate was centrifuged at $15,000 \times g$ for $45 \mathrm{~min}$ at $4^{\circ} \mathrm{C}$ and the supernatant was used for assay of the activity of SOD and catalase.

SOD activity was measured by the nitroblue tetrazolium (NBT) reduction method [15] using the Wako-SOD test (Wako Pure Chemical Industry, Co., Ltd., Osaka, Japan). Briefly, the extent of inhibition of the reduction in NBT was measured at $560 \mathrm{~nm}$ using a spectrophotometer. One unit of enzyme activity was defined as $50 \%$ inhibition of NBT reduction.

Catalase activity was measured as the hydrogen peroxide $\left(\mathrm{H}_{2} \mathrm{O}_{2}\right)$ reduction rate at $37^{\circ} \mathrm{C}$ and was assayed at $240 \mathrm{~nm}$ using a spectrophotometer [16]. The assay mixture consisted of $50 \mu \mathrm{l}$ of $1 \mathrm{M}$ Tris-HCl buffer containing $5 \mathrm{mM}$ EDTA ( $\mathrm{pH}$ 7.4), $900 \mu \mathrm{l}$ of $10 \mathrm{mM} \mathrm{H}_{2} \mathrm{O}_{2}, 30 \mu \mathrm{l}$ deionized water, and $20 \mu \mathrm{l}$ liver supernatant. Activity was calculated using a molar extinction coefficient of $7.1 \times 10^{-3} \mathrm{M}^{-1} \mathrm{~cm}^{-1}$. Catalase activity was measured by the amount of hydrogen peroxide split by catalase in 20,40 , or $60 \mathrm{~s}$ at $37^{\circ} \mathrm{C}$. The reactions were started by addition of the liver supernatant.

\section{Histological observation}

Livers were fixed in $10 \%$ formalin, processed with a graded mixture of ethanol and xylene and embedded in 


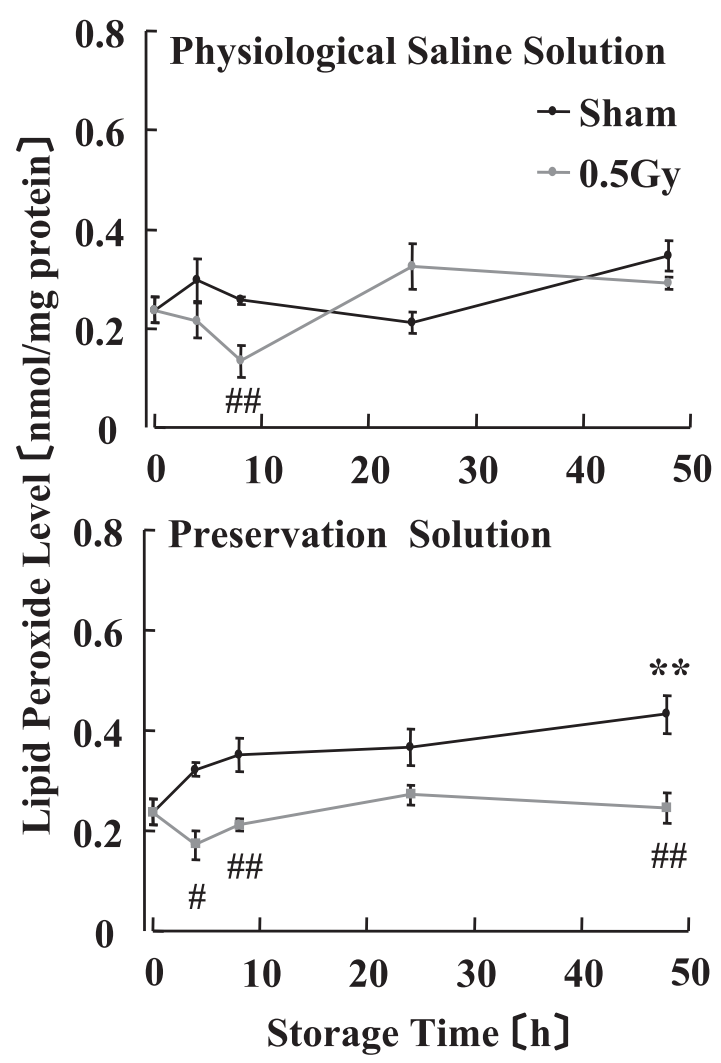

Fig. 1. Changes in lipid peroxide levels of mouse livers in preservation after $0.5 \mathrm{~Gy}$ irradiation. Mouse livers were preserved in physiological saline or preservation solution for the indicated number of hours following sham irradiation of irradiation of $0.5 \mathrm{~Gy}$ as indicated. Lipid peroxide levels were measured with a commercial assay kit. Each value indicates the mean \pm SEM. The number of mouse livers per experimental point is $5-10$. * $p<0.05$, $* * p<0.01, * * * p<0.001$ vs control, ${ }^{*} p<0.05,{ }^{\#} p<0.01$, ${ }^{\# \#} p<0.001$ vs sham irradiation (storage in physiological saline solution).

paraffin. The $6-\mu \mathrm{m}$ thick tissue sections were prepared and stained with hematoxylin-eosin (HE).

\section{Statistical analyses}

The data values are presented as the mean \pm standard error of the mean (SEM). The statistical significance of differences was determined by Student's $t$ test for comparison between two groups and Dunnet's tests for multiple comparisons where appropriate. Each experimental group consisted of 5-10 samples.

\section{Results}

Changes in the level of oxidative damage in mouse livers after irradiation

To determine changes in oxidative damage in mouse

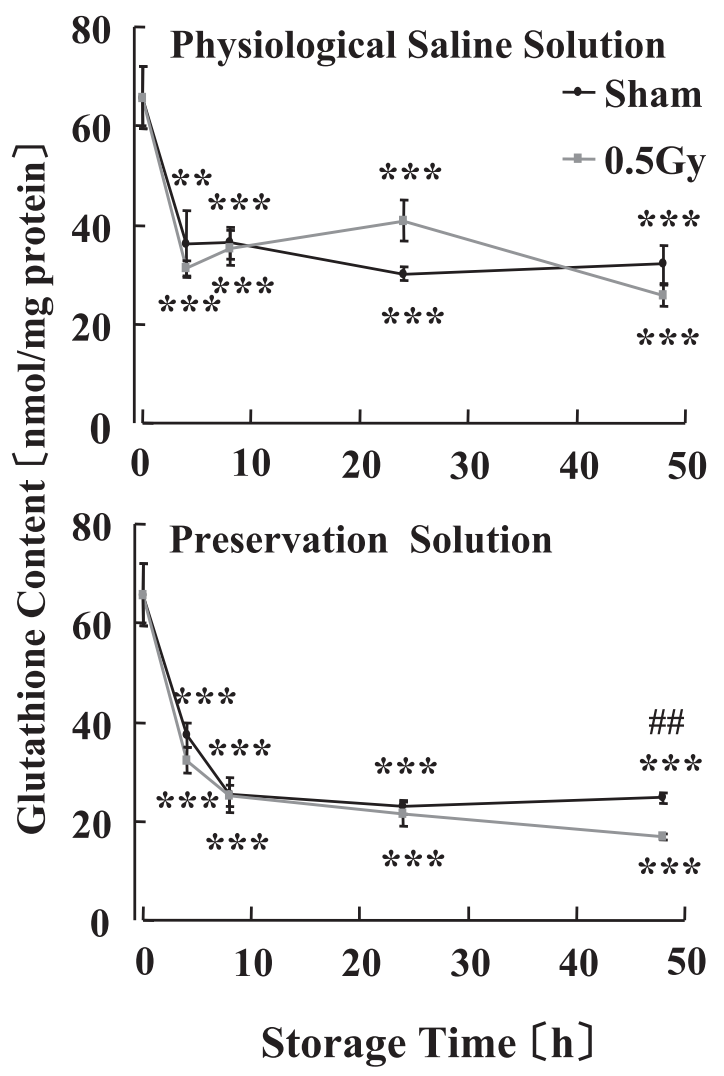

Fig. 2. Changes in total glutathione content of mouse livers in preservation after 0.5 Gy irradiation. Mouse livers were treated as described for Fig. 1 and glutathione content was measured by a spectrophotometric assay. The data, number of mouse livers and significance are as described for Fig. 1.

livers after irradiation various parameters of oxidative damage were assayed in livers that were stored in physiological saline or preservation solution following sham- or $\mathrm{X}$-irradiation. Lipid peroxide levels were significantly increased in mouse livers kept in cold storage for $48 \mathrm{~h}$ in preservation solution after sham irradiation. Lipid peroxide levels of mouse livers kept in cold storage for $8 \mathrm{~h}$ in saline solution, or for 4,8 , or $48 \mathrm{~h}$ in preservation solution, after $0.5 \mathrm{~Gy}$ irradiation were significantly lower than those after sham irradiation (Fig. 1).

The total glutathione content of mouse livers kept in cold storage for $4,8,24$, or $48 \mathrm{~h}$ in physiological saline or in preservation solution after sham or 0.5 Gy irradiation was significantly decreased. Total glutathione content of mouse livers kept in cold storage for $48 \mathrm{~h}$ in preservation solution after 0.5 Gy irradiation were significantly lower than those after sham irradiation (Fig. 2).

SOD activity of mouse livers kept in cold storage for 8 , 24 , or $48 \mathrm{~h}$ in saline solution after $0.5 \mathrm{~Gy}$ irradiation was significantly increased. The SOD activity of mouse livers kept for $24 \mathrm{~h}$ in cold storage in saline solution after $0.5 \mathrm{~Gy}$ 


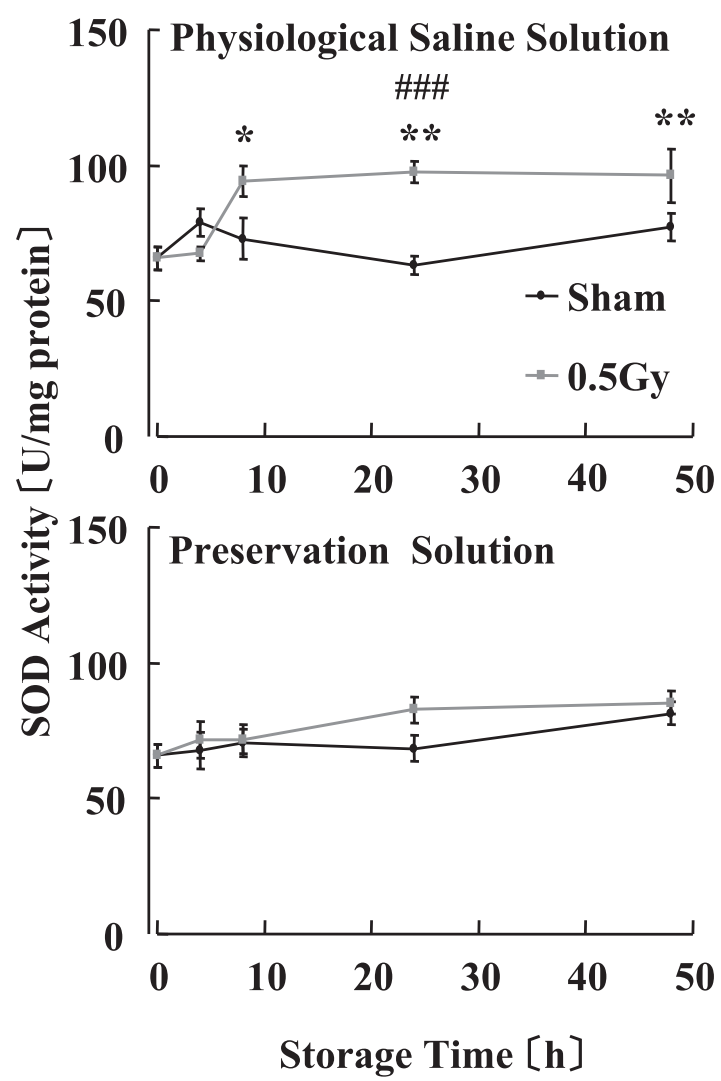

Fig. 3. Changes in SOD activity of mouse livers in preservation after 0.5 Gy irradiation. Mouse livers were treated as described for Fig. 1 and SOD activity was measured by a spectrophotometric assay. The data, number of mouse livers and significance are as described Fig. 1.

irradiation were significantly higher than those after sham irradiation (Fig. 3).

Catalase activity of mouse livers kept in cold storage for 4 or $24 \mathrm{~h}$ in saline solution after 0.5 Gy irradiation was significantly increased. The catalase activities of mouse livers kept for $24 \mathrm{~h}$ in cold storage in saline solution after 0.5 Gy irradiation were significantly higher than those after sham irradiation (Fig. 4). These data indicate that parameters of oxidation in mouse livers are highly responsive to irradiation and storage conditions.

Changes in oxidative damage levels in mouse livers kept in cold storage for $24 \mathrm{~h}$ after irradiation

We next examined in further detail the effect of storage of livers in preservation or saline solution for $24 \mathrm{~h}$ following irradiation on parameters of oxidative damage. The lipid peroxide level of mouse livers stored in preservation solution after 0.25 Gy irradiation was significantly lower than that after sham irradiation. The levels of lipid peroxide in livers kept in preservation solution after sham irradiation were significantly higher than those kept in saline solution

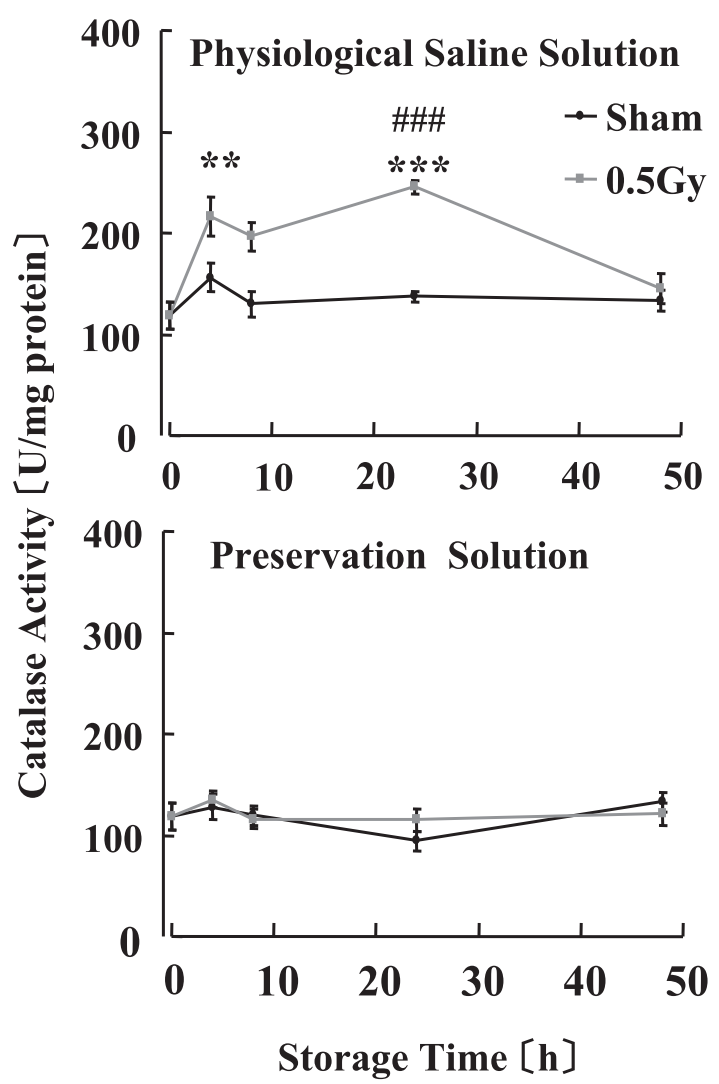

Fig. 4. Changes in catalase activity of mouse liver in preservation after $0.5 \mathrm{~Gy}$ irradiation. Mouse livers were treated as described for Fig. 1 and catalase activity was measured by a spectrophotometric assay. The data, number of mouse livers and significance are as described Fig. 1.

(Fig. 5A).

The total glutathione content of mouse livers stored in preservation solution after sham, 0.5 , or 1.0 irradiation was significantly lower than that in saline solution (Fig. 5B).

The SOD activity of mouse livers stored in preservation solution after $0.25,1.0$, or 5.0 Gy irradiation, or in saline solution after $0.25,0.5$, or 5.0 Gy irradiation, was significantly higher than that after sham irradiation. SOD activity in livers stored in preservation solution after 1.0 Gy irradiation was significantly higher than that in saline solution (Fig. 5C).

The catalase activity of mouse livers, stored in preservation solution after 1.0 Gy irradiation, or in saline solution after 0.25 or 0.5 Gy irradiation, was significantly higher than that after sham irradiation. Catalase activity of mouse livers stored in preservation solution after 1.0 Gy irradiation was significantly higher than that in saline solution. Moreover, catalase activity in livers stored in preservation solution after sham, 0.25 , or $0.5 \mathrm{~Gy}$ irradiation was significantly lower than that in saline solution (Fig. 5D).

These data suggest that the type of solution in which livers 

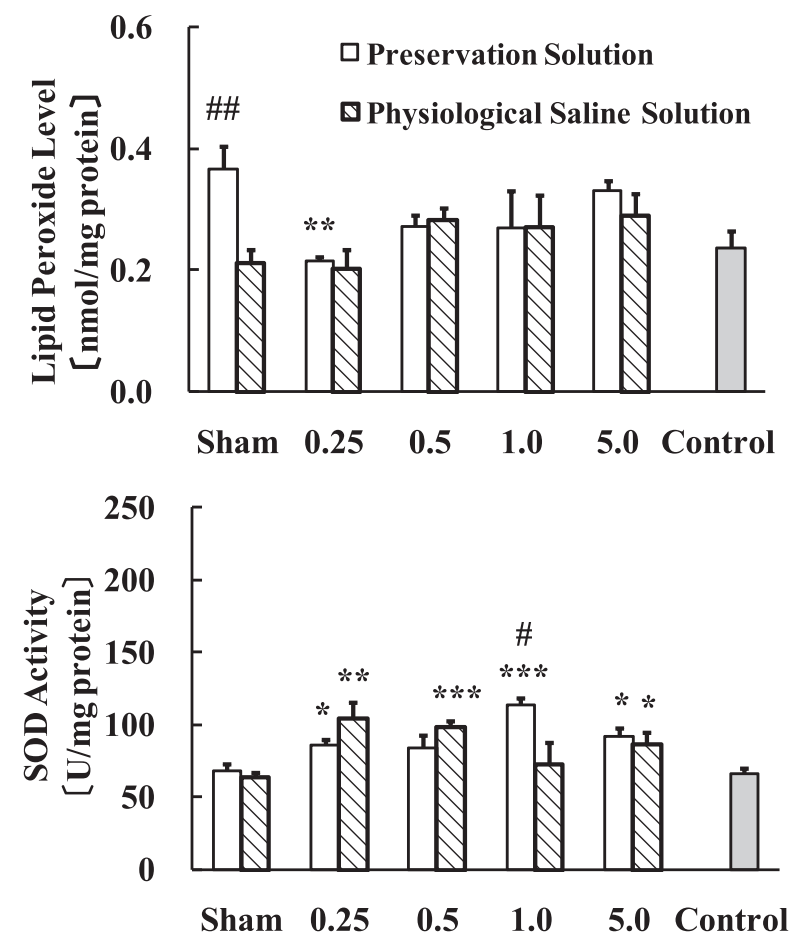
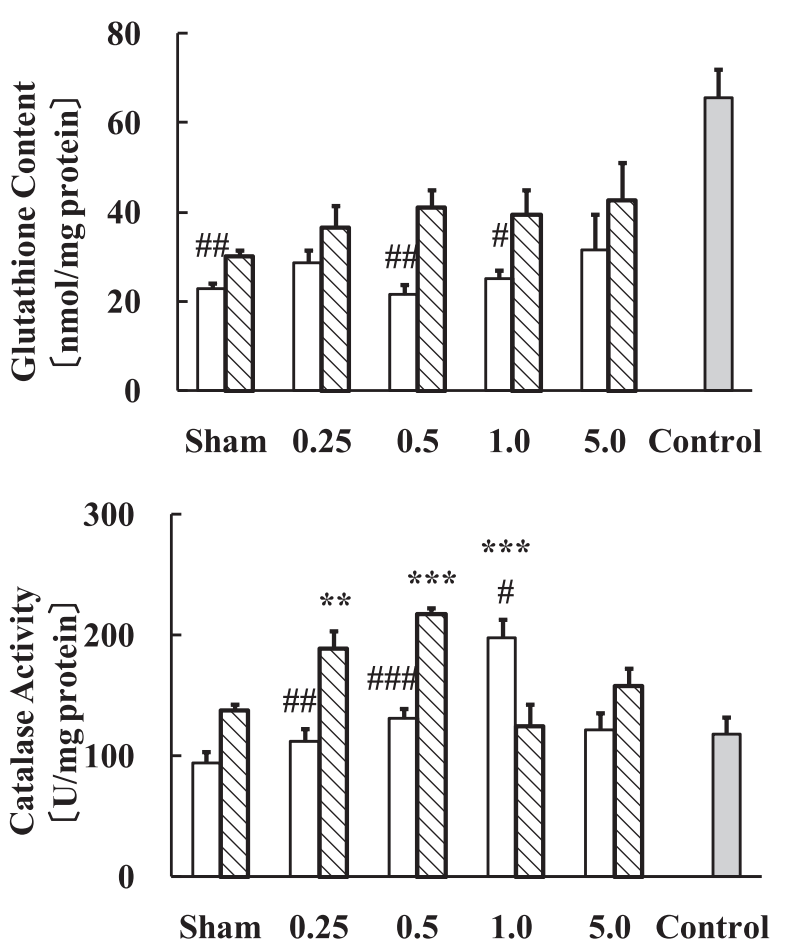

Doses of X-rays [Gy]

Fig. 5. Changes in (A) lipid peroxide level, (B) glutathione content, and activities of (C) SOD and (D) catalase in mouse liver preserved for $24 \mathrm{~h}$ following irradiation. Mouse livers were treated as described for Fig. 1 and glutathione content was measured by a spectrophotometric assay. Each value indicates the mean \pm SEM. The number of mouse livers per experimental point is $5-10 .{ }^{*} p<0.05,{ }^{* *} p<0.01,{ }^{* * *} p<0.001$ vs each solution by sham irradiation, ${ }^{\#} p<0.05,{ }^{*} p<0.01$, \#\#\# $p<0.001$ vs physiological saline solution.

are stored plays a critical role in the level of oxidative damage in livers during storage following irradiation.

\section{Histological examination}

Histological changes in mouse livers following irradiation and storage in saline or preservation solution were examined. Histological examination of hepatocytes kept in cold storage in saline solution following irradiation indicated that the hepatocytes were vacuolated under these conditions. In contrast, no vacuole formation was observed in hepatocytes kept in cold storage in preservation solution following irradiation (Fig. 6).

\section{Discussion}

The findings of this study provide important insights concerning oxidative changes in liver following preservation in different solutions after irradiation. Oxidative changes appear to play a critical role in ischemic injury.

The fact that SOD and catalase are just as effective as antioxidants when administered just before re-oxygenation as when administered before anoxia confirms that ischemic injury occurs primarily at re-oxygenation, and that this injury is mediated by a process dependent upon superoxide formation. Allopurinol, which inhibits xanthine oxidase synthesis, substantially reduces ischemic injury because xanthine is major source of free radical generation within endothelial cells at reoxygenation [17]. The preservation solution contains glutathione, allopurinol, and adenosine that have been added to alleviate ischemia-reperfusion injury after transplantation.

Low-dose irradiation promotes a small induction of ROS in vivo and induces the production of antioxidant substances including SOD, catalase and glutathione, in various organs [18]. Previous studies have shown that low-dose Xirradiation or low-level radon inhalation enhances antioxidative and immune functions and reduces oxidative damage such as ischemia-reperfusion injury and liver damage [13, 19-22]. In addition, it has been reported that SOD and catalase improve cell viability following ischemiareperfusion injury after liver transplantation [23]. Thus, it is highly possible that low-dose irradiation inhibits ischemiareperfusion injury after liver transplantation.

We examined changes in antioxidative function in liver 

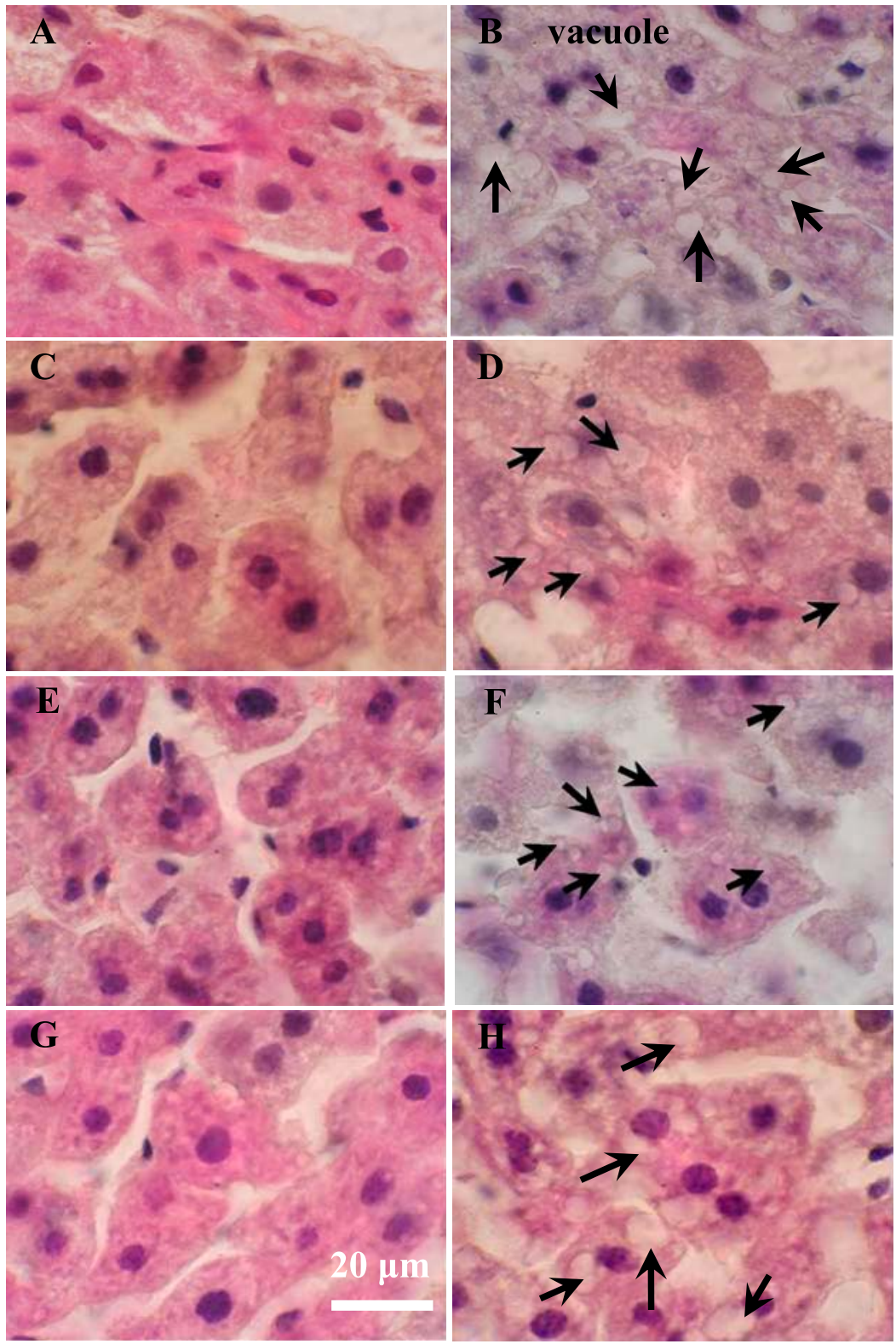

Fig. 6. Histological changes in mouse liver in preservation after irradiation. Mouse livers were examined histologically following preservation under the following conditions. A) Control, B) Cold storage for $4 \mathrm{~h}$ in physiological saline solution after sham irradiation, C) Cold storage for $24 \mathrm{~h}$ in preservation solution after sham irradiation, D) Cold storage for $24 \mathrm{~h}$ in physiological saline solution after sham irradiation, E) Cold storage for $48 \mathrm{~h}$ in preservation solution after sham irradiation, F) Cold storage for $48 \mathrm{~h}$ in physiological saline solution after sham irradiation, G) Cold storage for $24 \mathrm{~h}$ in preservation solution after $1.0 \mathrm{~Gy}$ irradiation, and $\mathrm{H}$ ) Cold storage for $24 \mathrm{~h}$ in physiological saline solution after $1.0 \mathrm{~Gy}$ irradiation. The arrow shows vacuole formation in the liver. The length of the scale bar is $20 \mu \mathrm{m}$. For all figures HE staining was used.

grafts following low-dose $\mathrm{X}$-irradiation immediately after exenteration. Our results show that under these conditions SOD and catalase of mouse livers that are stored in saline solution after 0.5 Gy irradiation, or in preservation solution after 1.0 Gy irradiation, are significantly increased and that the peak of enhancement of antioxidative function is around $24 \mathrm{~h}$ after irradiation. However, a previous report suggested that the peak of enhancement of antioxidative function was around $4 \mathrm{~h}$ after low-dose irradiation [24]. The difference in the time of peak enhancement of antioxidative function between our study and the previous study are probably due to differences in the conditions of irradiation and preservation.

Catalase activity in mouse livers kept in cold storage for $24 \mathrm{~h}$ in saline solution after 0.25 or 0.5 Gy (but not $1.0 \mathrm{~Gy}$ ) irradiation was increased compared to sham control. In 
this study, we used a preservation solution which contained glutathione, allopurinol, and adenosine. Glutathione and allopurinol prevent cytotoxic injuries from oxygen free radicals. Adenosine provides a metabolic substrate for the regeneration of ATP at the initial period of reperfusion. These findings suggest that the enhancement of antioxidative function in samples that were stored in preservation solution was significantly higher than that in saline solution because a small volume of ROS, induced in vivo by excess irradiation, may be neutralized by glutathione which is contained in the preservation solution.

There was no correlation between the SOD or catalase activity and the total glutathione content. Glutathione is synthesized from L-glutamate, L-cysteine, adenine triphosphate (ATP), and glycine. However, the ATP level of liver tissue is gradually decreased during preservation in the cold [25-28]. The decline of glutathione during ischemia may reflect an inhibition of glutathione synthesis since three equivalents of ATP are required for the synthesis of one glutathione [25-28].

It is well known that peroxidation of unsaturated fatty acids of cell membrane phospholipids is accompanied by alterations in membrane structure and function. Lipid peroxidation affects the physical properties of membranes, including membrane fluidity, and normal metabolism and electron transport [29, 30]. The lipid peroxide level gradually increases during preservation of liver graft in the UW solution [31]. Our data showed that the lipid peroxide level in livers stored in preservation solution was significantly increased only at $48 \mathrm{~h}$ of storage. Furthermore, that there was no marked difference in lipid peroxide levels between sham and X-irradiated livers stored in physiological solution. In contrast, lipid peroxide levels of the 0.5 Gy irradiated group were lower than those of the sham irradiated group following 4, 8 , or $48 \mathrm{~h}$ of storage in preservation solution. Previous studies have shown that low-dose X-irradiation activates antioxidant function, including the activity of SOD and catalase, and decreases the level of lipid peroxide [19, 20]. However, in this study, the activity of SOD and catalase did not appear to correlate with the prevention of lipid peroxidation either in saline solution or in preservation solution as we have previously reported [19, 20]. One activity of glutathione is the reduction of lipid peroxide. Our results showed that the total glutathione content of mouse livers maintained in cold storage for $48 \mathrm{~h}$ in preservation solution after $0.5 \mathrm{~Gy}$ irradiation was significantly lower than that after sham irradiation. These findings indicate that the consumption of glutathione decreases the total level of glutathione in liver grafts and inhibits lipid peroxidation during storage.

In addition to measurement of the biochemical activity of molecules involved in oxidation, we also examined histological changes in hepatocytes after storage. These data indicated that hepatocytes stored in saline solution were vacuolated following irradiation at all intensities. In contrast, hepatocytes stored in preservation solution were not vacuolated following any irradiation. These results suggest that storage of liver in saline solution is of no benefit for future use of the liver for transplantation in spite of the increase in antioxidative function.

The data presented in this study provide an essential basis for future studies aimed at determination of the possibility of remission of ischemia-reperfusion injury in livers after transplantation.

\section{Acknowledgments}

The authors thank Dr. Soichiro Nose (Okayama Saiseikai General Hospital) for his technical advice.

\section{Abbreviations}

UW, University of Wisconsin; ROS, reactive oxygen species; XOD, xanthine oxidase; SOD, superoxide dismutase; GPx, glutathione peroxidase; GR, glutathione reductase; MDA, malondialdehyde; PBS, phosphate buffer; EDTA, ethylendiaminetetraacetic acid; NBT, nitroblue tetrazolium; $\mathrm{H}_{2} \mathrm{O}_{2}$, hydrogen peroxide; HE, hematoxylin-eosin; SEM, standard error of mean; ATP, Adenine triphosphate; AMP, adenosine monophosphate; GSSG, oxidized glutathione; $\mathrm{CCl}_{4}$, carbon tetrachloride.

\section{References}

[1] Mehta, N. and Mirza, D.F.: Liver transplantation. Surgery, 25, 42-46, 2006.

[2] Clavein, P.A., Harvey, P.R., and Strasberg, S.M.: Preservation and reperfusion injuries in liver allografts. An. Overview and synthesis of current studies. Transplantation, 53, 957978, 1992.

[3] Fellstrom, B., Akuyrek, L.M., Backman, U., Larsson, E., Melin, J., and Zezina, L.: Postischemic reperfusion injury and allograft arteriosclerosis. Transplant. Proc., 30, 42784280, 1998.

[4] Neville, V.J., Ralf, S., Susanne, L., Kerstin, C., Jon, M., Paul, K.V., Derek, G.D.W., James, H.S., and Folkert, O.B.: Preservation of the canine liver for 24-48 hours using simple cold storage with UW solution. Transplantation, 46, 517-522, 1998.

[5] McCord, J.M.: Oxygen-derived free radicals in postischemic tissue injury. N. Engl. J. Med., 312, 159-163, 1985.

[6] Braunwald, E. and Lonar, R.A.: Myocardial reperfusion. A double-edged sword? J. Clin. Invest., 16, 1713-1719, 1985.

[7] Mizuta, T., Saito, A., Kawano, N., Nagao, T., and Morioka, Y.: The beneficial effect of superoxide dismutase on the rat liver graft. Jpn. J. Surg., 19, 208-212, 1989.

[8] Kojima, S., Matsuki, O., Nomura, T., Kubodera, A., Honda, 
Y., Honda, S., Tanooka, H., Wakasugi, H., and Yamaoka, K.: Induction of mRNAs for glutathione synthesis-related proteins in mouse liver by low doses of $\gamma$-rays. Biochem. Biophys. Acta, 1381, 312-318, 1998.

[9] Kojima, S., Matsuki, O., Nomura, T., Shimura, N., Kubodera, A., Yamaoka, K., Tanooka, H., Wakasugi, H., Honda, Y., Honda, S., and Sakai, T.: Localization of glutathione and induction of glutathione synthesis-related proteins in mouse brain by low-doses $\gamma$-rays. Brain Res., 808, 262-269, 1998.

[10] Jain, A., Mårtensson, J., Stole, E., Auld, P.A., and Meister, A.: Glutathione deficiency leads to Mitochondrial damage in the brain. Proc. Natl. Acad. Sci. USA, 88, 1913-1917, 1991.

[11] Martensson, J., Jain, A., Stole, E., Frayer, W., Auld, P.A.M., and Meister, A.: Induction of glutathione synthesis in the new born rat: a model of endogenously produced oxidative stress. Proc. Natl. Acad. Sci. USA, 88, 9360-9364, 1991.

[12] Kojima, S., Matsuki, O., Kinoshita, I., Gonzalez, T.V., Shimura, N., and Kubodera, A.: Dose small-dose $\gamma$-ray radiation induce endogenous antioxidant potential in vivo? Biol. Pharm. Bull., 20, 601-604, 1997.

[13] Kataoka, T., Mizuguchi, Y., Yoshimoto, M., Taguchi, T., and Yamaoka, K.: Inhibitory effects of prior low-dose Xirradiation on ischemia-reperfusion injury in mouse paw. $J$. Radiat. Res., 48, 505-513, 2007.

[14] Bradford, M.M.: A rapid and sensitive method for the quantitation of microgram quantities of protein utilizing the principle of protein-dye binding. Anal. Biochem., 72, 248254, 1976.

[15] Robert, L.B., Suzanne, K.M., Jacqueline, D., and Richard, B.J. Jr.: The role of superoxide anion and hydrogen peroxide in phagocytosis-associated oxidative metabolic reactions. $J$. Clin. Invest., 56, 571-576, 1975.

[16] Aebi, H., Wyss, S.R., Scherz, B., and Gross, J.: Properties of erythrocyte catalase from homozygotes and heterozygotes for Swiss-type acatalasemia. Biochem. Genet., 14, 791-807, 1976.

[17] Oyanagui, Y., Sato, S., and Okajima, T.: Suppression of ischemic paw oedema in mice, rats and guinea pigs by superoxide dismutases from different sources. Free Radic. Res. Commun., 4, 385-396, 1988.

[18] Yamaoka, K.: Activation of antioxidant system by low dose radiation and its applicable possibility for treatment of reactive oxygen species-related diseases. J. Clin. Biochem. Nurt., 39, 114-133, 2006.

[19] Yamaoka, K., Kataoka, T., Nomura, T., Taguchi, T., Wang, D.H., Mori, S., Hanamoto, K., and Kira, S.: Inhibitory effects of prior low-dose irradiation on carbon tetrachloride-induced hepatopathy in acatalasemic mice. J. Radiat. Res., 45, 89-95, 2004.
[20] Kataoka, T., Nomura, T., Wang, D.H., Taguchi, T., and Yamaoka, K.: Effects of post low-dose X-ray irradiation on carbon tetrachloride-induced acatalasemic mice liver damage. Physiol. Chem. Phys. Med. NMR, 37, 109-126, 2005.

[21] Kataoka, T., Mizoguchi, Y., Notohara, K., Taguchi, T., and Yamaoka, K.: Histological changes in spleens of radiosensitive and radio-resistant mice exposed to low-dose X-ray irradiation. Physiol. Chem. Phys. Med. NMR, 38, 21-29, 2006.

[22] Kataoka, T., Aoyama, Y., Sakoda, A., Nakagawa, S., and Yamaoka, K.: Basic study on biologic effects of thoron and thermal therapy. Physiol. Chem. Phys. Med. NMR, 38, 8592, 2006.

[23] Tanaka, J., Malchesky, P.S., Omokawa, S., Goldcamp, J.B., Harasaki, H., Vogt, D.P., Broughan, T.A., and Nosé, Y.: Effects of prostaglandin I2, superoxide dismutase, and catalase on ischemia-reperfusion injury in liver transplantation. ASAIO Trans., 36, 600-603, 1990.

[24] Yamaoka, K., Edamatsu, R., and Mori, A.: Increased SOD activities and decreased lipid peroxide levels induced by low dose X irradiation in rat organs. Free Radic. Biol. Med., 11, 299-306, 1991.

[25] Ohkohchi, N., Sakurada, M., Koyamada, M., Katoh, H., Koizumi, M., Hirano, T., Orii, T., Kanno, M., Terashima, T., Satoh, K., Satomi, S., Taguchi, Y., and Mori, S.: The importance of preservation of sinusoidal endothelial cell injury during cold preservation of liver graft. Tohoku J. Exp. Med., 174, 317-331, 1994.

[26] Scaduto, R.C. Jr., Gattone, V.H. 2nd, Grotyohann, L.W., Wertz, J., and Martin, L.F.: Effect of an altered glutathione content on renal ischemic injury. Am. J. Physiol., 255, F911F921, 1988.

[27] Stephan, O.S., Lee, W.G., Lousi, F.M., and Russell, C.S. Jr.: Glutathione catabolism by ischemia rat kidney. Am. J. Physiol., 258, F1547-F1553, 1990.

[28] Wefers, H. and Sies, H.: Oxidation of glutathione by the superoxide radical to the disulfide and the sulfonate yielding singlet oxygen. Eur. J. Biochem., 137, 29-36, 1983.

[29] Watanabe, H., Kobayashi, A., Yamamoto, T., Suzuki, S., Hayashi, H., and Yamazaki, N.: Alteration of human erythrocyte membrane fluidity by oxygen-derived free radicals and calcium. Free Radic. Biol. Med., 9, 507-514, 1990.

[30] Papa, S. and Skulachev, V.P.: Reactive oxygen species, mitochondria associated with aging. Mol. Cell Biochem., 174, 305-319, 1997.

[31] Serrano, E., Diaz, J., Acosta, F., Palenciano, C.G., Parrilla, P., and Carbonell, L.F.: Oxidative stress during ischemiareperfusion in liver transplantation. Transplant. Proc., 32, $2651,2000$. 